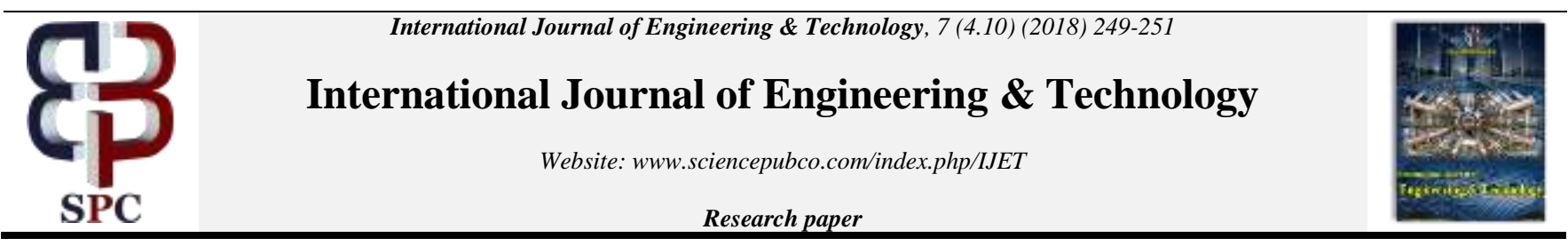

\title{
Construction of Reverse Super Edge Magic Total Graphs
}

\author{
U.Masthan Raju ${ }^{1}$ and S.Sharief Basha ${ }^{* 2}$ \\ ${ }^{1}$ Research Scholar, Rayalaseema University, Kurnool, Andhra Pradesh, India. \\ ${ }^{2}$ School of Advanced Sciences, VIT, Vellore-632 014, Tamil Nadu, India \\ *Corresponding author E-mail: shariefbasha.s@vit.ac.in
}

\begin{abstract}
In this paper, we investigate the adjacency matrix of reverse super edge magic vertex graph and use this graph to construct other reverse super edge magic graphs with the same edge weight set. Additionally, by combining known reverse super edge magic labelled graphs, we give a construction for a new reverse super edge magic graph
\end{abstract}

Keywords: super edge magic total labeling, super edge magic total graphs, Reverse Super edge magic labeling, Reverse super edge magic graphs.

\section{Introduction}

Let $\mathrm{G}$ be a finite simple undirected graph. The set of vertices and edges of a graph $\mathrm{G}$ will be denoted by $\mathrm{V}(\mathrm{G})$ and $\mathrm{E}(\mathrm{G})$, respectively, $v=|V(G)|$ and $e=|E(G)|$. For simplicity, we denote $\mathrm{V}(\mathrm{G})$ by $\mathrm{V}$ and $\mathrm{E}(\mathrm{G})$ by $\mathrm{E}$.

A labeling of a graph $G$ is a mapping that carries a set of graph elements into a set of numbers (usually positive integers), called labels. Kotzig and Rosa in 1970 introduced edge magic total labeling [5].

An edge magic total (EMT) labeling is a one-to-one mapping $f$ from $\mathrm{VuE}$ onto the integers $1,2, \ldots, \mathrm{v} \cdot+\mathrm{e}$ with the property that for every $(x, y)$ in $E, f(x)+f(y)+f(x y)=k$ for some constant $k$. A graph that has an edge magic total labeling is called an edge magic total graph. An edge magic total labeling is called a super edge magic total (SEMI) labeling if $f(V)=\{1,2, \ldots, v\}$ and a graph that has SEMT labeling is called a SEMT graph. Research in SEMT labeling has been particularly popular during the last decade. For details, see the Gallian's dynamic survey [4]. There are many open problems, some of which will be listed in the conclusion of this paper.

S.Venkata Ramana etal [13] introduced the concept of reverse super edge-magic labeling of $\mathrm{G}$. A one to one map $\mathrm{f}$ from $V \cup E$ onto the integers $\{1,2, \ldots . v+\mathcal{E}\}$ is a reverse edgemagic labeling if there exists a constant $\mathrm{k}$ so that for any edge $\mathrm{xy}$, $\mathrm{f}(\mathrm{xy})-\{\mathrm{f}(\mathrm{x})+\mathrm{f}(\mathrm{y})\}=\mathrm{k}$. The constant $\mathrm{k}$ is called the reverse edgemagic number for $\mathrm{f}$. A reverse edge-magic labeling $\mathrm{f}$ is called reverse super edge-magic if $\mathrm{f}(\mathrm{V})=\{1,2,3, \ldots \ldots, v\}$ and $\mathrm{f}(\mathrm{E})=\{v+1, v+2, v+3, \ldots \ldots \ldots . . v+\mathcal{E}\}$. a graph $\mathrm{G}$ is called reverse super edge-magic if there exists a reverse super edgemagic labeling of $\mathrm{G}$.

Concerning SEMT graph, researchers usually concentrate on some specific class of families of graphs, such as trees, cycles, bipartite graphs, friendship graphs, wheels, generalised Petersen graphs. See $[2,3,5,6,7,11]$. In this paper, we use the adjacency matrix of a known SEMT graph to construct other labeled graphs with the same edge-weights set. Additionally, we give a construction of new graphs by combining several graphs that have SEMT. Adjacency matrix methods have been used to generate a reverse super edge magic graph in [12]. However, this is the first time that adjacency matrices are used to generate SEMT graphs.

\section{Adjacency Matrix}

Let $\mathrm{G}=(\mathrm{V}(\mathrm{G}), \mathrm{E}(\mathrm{G}))$ be a graph and $\mathrm{f}$ be an EAV labeling of $\mathrm{G}$. Let $V=\left\{X_{1}, X_{2}, \ldots, X_{v}\right\}$ be the set of vertices in $G$ with the labels $\{1,2, \ldots, v\}$. Let $A$ be an adjacency matrix of $G$, then the rows and columns of A can be labeled using $1,2, \ldots, \mathrm{v}$. A is symmetric and every skew diagonal (diagonal of A which is traversed in the "northeast" direction) line of matrix A has at most two "I" elements. The weights set $\{f(x)+f(y): x, y \in V\}$ generates a consecutive integers $\mathrm{a}, \mathrm{a}+1, \ldots, \mathrm{a}+\mathrm{e}-1$ for some positive integer $\mathrm{a}$. The weight $f(x)+f(y)$ is the same as the sum of labels of vertices on skew diagonal adjacency matrix that has "1" element.

A graph that has an EAV labeling and has the maximum possible number of edges is called maximal EAV graph. If $\mathrm{G}$ has a maximal EAV labeling then $\mathrm{a}=3$. Enomoto et al. [2] proved that the maximal number of edges in a SEMT graph is $2 \mathrm{v}-3$.

Let $\mathrm{A}=\left(\mathrm{a}_{\mathrm{ij}}\right)$ be an adjacency matrix of a maximal EAV graph $\mathrm{G}$. We can easily see that $\left\{\mathrm{a}_{\mathrm{lj}}: \mathrm{a}_{\mathrm{lj}} \neq 0, \mathrm{j}=1, \ldots, \mathrm{v}\right\}=\mathrm{v}-1$ and $\left\{\mathrm{a}_{\mathrm{il}}: \mathrm{a}_{\mathrm{il}}\right.$ $\neq 0, \mathrm{i}=1, \ldots, \mathrm{v}\}=\mathrm{v}-1$. Note that $\mathrm{a}_{\mathrm{vv}}$ is counted twice. Thus the maximal width of the band of non-empty skew diagonal line is $2 \mathrm{v}$ -3 .

Let $\mathrm{A}$ be the adjacency matrix of an EAV graph $\mathrm{G}$ of order v. If we move the element "1" of A along the skew-diagonal line, then this matrix is an adjacency matrix of an EAV graph that has the same weights set as A. Two graphs $\mathrm{G}$ and $\mathrm{G}^{*}$ are EA V-equivalent if $G^{*}$ is obtained by the previous technique of moving the "1" element from G. Note that EAV-equivalent graphs are not necessarily isomorphic with respect to the graph structure and/or to the vertex labels.

Figure 1 shows an example of generating a new maximal EAV graph from an old one. Graph

$\mathrm{G}^{*}$ is obtained from graph $\mathrm{G}$ by moving the element "I" from position $(1,4)$ to position $(2,3)$ in the same skew-diagonal line.

Baca et al. [1] proved that if $G$ has an EAV labeling then $G$ has SEMT labeling. Thus, in this paper, we consider an adjacency 
matrix of an EAV graph. Another known result for maximal RSEM labeling is given in the next section.

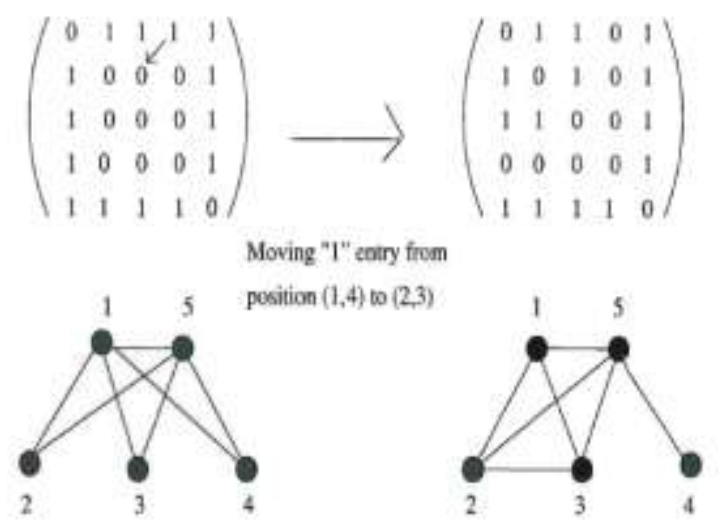

Figure 1: Generating a new EAV grapts.

\section{Maximal RSEM Labeling}

Figure 2 gives all maximal EAV-equivalent graphs with the one in Fig 1. Using the computer search we can find all possibilities of maximal EAV-equivalent graph from a given EAV graph with small order. Table 1 gives the result of the searching. Sugeng and Miller in [12] showed that the number of maximal EAVequivalent (both connected and disconnected) graphs with size $\mathrm{v}$ is

- $\left(\frac{v-3}{2} !\right)^{4}\left(\frac{v-1}{2}\right)^{3}$, for $v$ odd

- $\left(\frac{v-2}{2} !\right)^{4}\left(\frac{v}{2}\right)$, for v even.
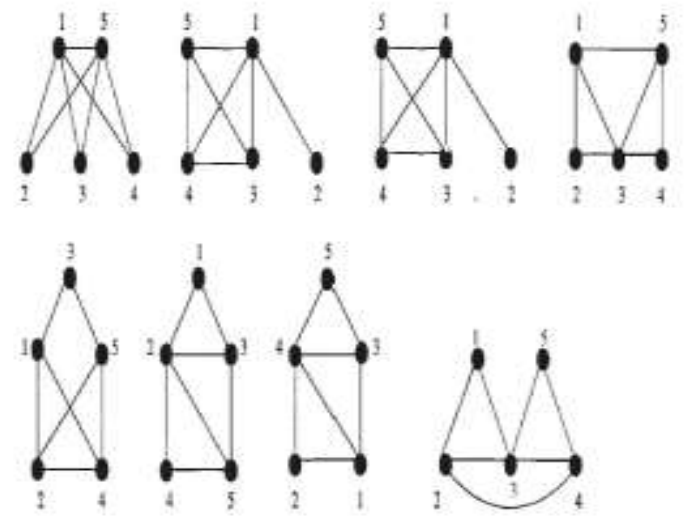

Figure 2: Maximal BAV exuivalent graphs on 5 rertios

MacDougall and Wallis [9] studied SEMT maximal graphs. They called SEMT a strong edge-magic-total labeling. They proved the following propositions:

\begin{tabular}{|c|c|c|}
\hline$v$ & Connected EAV-equivalent & Disconnected EAV-equivalent \\
\hline 5 & 8 & 0 \\
\hline 6 & 48 & 0 \\
\hline 7 & 420 & 12 \\
\hline 8 & 4896 & 288 \\
\hline
\end{tabular}

Table 1: Maximal EAV-equivalent graphs on $v$ vertices.
Proposition 1 [9] Any SEMT labeling for a graph of order v can be obtained from any other by a sequence of single edge replacements.

This proposition is the same as our technique of moving the "1" element along the skew diagonal line of the adjacency matrix of a EAV graph.

Proposition 2 [9] Every maximal SEMT graph of order $v$ can be extended to one of order $\mathrm{v}+1$.

Proposition 3 can be generalized to the following theorem, giving a new SEMT graph from two known maximal SEMT graphs.

Theorem 1 [9] Let Gl and G2 be any maximal SEMT graphs of order $\mathrm{v}$ and $\mathrm{w}$, respectively. Then there are SEMT graphs of orders $v+w-2, v+w-1$, and $v+w$, each of which contains $G_{1}$ and $\mathrm{G}_{2}$ as induced sub graphs.

Considering the new maximal SEMT graph $\mathrm{G}$ with order $\mathrm{v}+\mathrm{w}$ like in the above theorem,

Observation 1 If $\mathrm{G}_{1}$ and $\mathrm{G}_{2}$ are maximal SEMT graphs order $\mathrm{v}$ and $w$ respectively, then we can construct a new maximal graph $\mathrm{G}$ with order $\mathrm{v}+\mathrm{w}$.

Next, we give new results on maximal SEMT labeling of regular graph.

Proposition 3 If an r-regular graph $\mathrm{G}$ is a maximal SEMT graph then the number of vertices $v$ is equal to 2,3 or 6 and

- if $\mathrm{v}=2$ then $\mathrm{r}=1$, or

- if $\mathrm{v}=3$ then $\mathrm{r}=2$, or

- if $v=6$ then $r=3$.

Proof. If $G$ is an r-regular maximal SEMT labeling then $\frac{r v}{2}=2 v-3$. It follows that $\mathrm{v} / 6$. Thus, $\mathrm{v}$ is equal to 2,3 or 6 .

The 1-regular graph with two vertices is $\mathrm{K}_{2}$ and the 2-regular graph with three vertices is cycle $\mathrm{C}_{3}$. It is known that $\mathrm{K}_{2}$ and $\mathrm{C}_{3}$ are SEMT graphs. Figure 3 gives EAV 3-regular graph on 6 vertices.
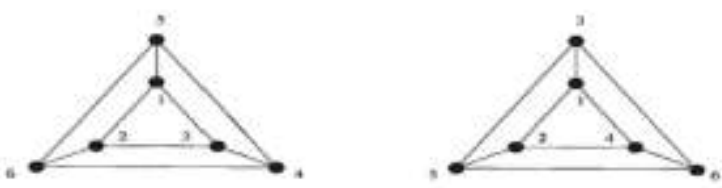

Figure 3: Maximal EAV 3-tegular graph on 6 verticent

\section{Non-MAXINLAL RSEM Graph}

In this section, we show how the adjacency matrix of an EAV graph can be used for manipulating a given non-maximal RSEM graph.

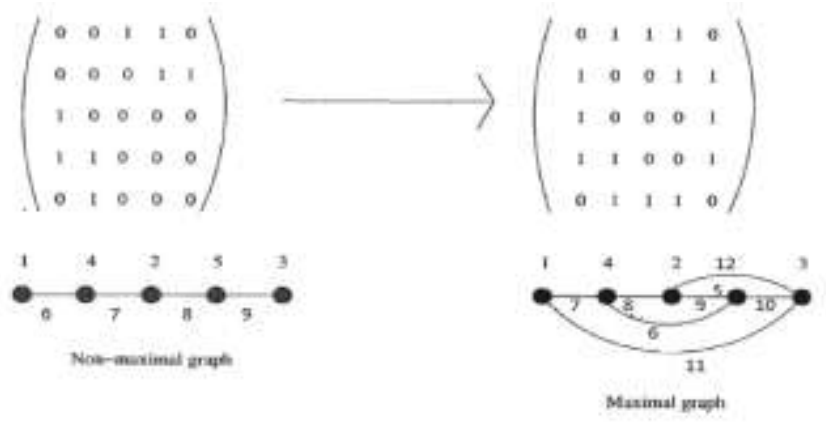

Figure 4: Expanding non-maximal RSEM graph on 5 vertices.

Theorem 2

Any non-maximal RSEM graph can be extended to a maximal RSEM graph.

\section{Proof}

If $\mathrm{G}$ is a non-maximal RSEM graph of order $\mathrm{v}$, then its adjacency matrix A has $\mathrm{v}$ rows and $\mathrm{v}$ columns but only $\mathrm{p}<2 \mathrm{v}-3$ non-empty 
skew-diagonal lines. Putting element "1" in $2 \mathrm{v}-3-\mathrm{p}$ empty skew-diagonal lines, we obtain a maximal RSEM graph.

Since the composition of edge in the graph has changed, then the edge labels for the new graph will also change. Figure 4 illustrates a maximal RSEM labeling extending a non maximal RSEM graph of order 5. We can see that $\mathrm{P}_{5}$ is not a maximal RSEM graph. It has only 4 edges. To extend $\mathrm{P}_{5}$ to a maximal RSEM graph, we need 3 more edges.

\section{Theorem 3}

Let $\mathrm{G}_{1}$ and $\mathrm{G}_{2}$ be any non-maximal RSEM graphs of order $\mathrm{v}$ and w respectively.

Then there exists an RSEM graph of order $\mathrm{v}+\mathrm{w}$ which contains $\mathrm{G}_{1}$ and $\mathrm{G}_{2}$ as induced sub graphs. The minimum number of additional edges needed is $2 \mathrm{v}-1+\min \left\{\mathrm{wt}\left(\mathrm{e}_{\mathrm{i}}\right): \mathrm{e}_{\mathrm{i}} \in \mathrm{E}\left(\mathrm{G}_{2}\right)\right\}-$ $\max \left\{w t(e j): e_{j} \in E\left(G_{2}\right)\right\}$.

\section{Proof:-}

Note that the weight of an edge xy under a labeling a is wt(xy) = $a(x y)-\{a(x)+a(y)\}$. Let $G_{1}$ and $G_{2}$ be non-maximal RSEM graphs of order $v$ and $w$ respectively, and with number of edges $e$ and $f$, respectively. Let $\mathrm{V}\left(\mathrm{G}_{1}\right)=\left\{\mathrm{x}_{\mathrm{l}}, \mathrm{x}_{2}, \ldots, \mathrm{x}_{\mathrm{v}}\right\}$ and $\mathrm{V}\left(\mathrm{G}_{2}\right)=\left\{\mathrm{y}_{1}, \mathrm{y}_{2}, \ldots\right.$, $\left.\mathrm{y}_{\mathrm{w}}\right\}$. Label the vertices in $\mathrm{G}_{1}$ and $\mathrm{G}_{2}$ as $\mathrm{a}\left(\mathrm{x}_{\mathrm{i}}\right)=\mathrm{i}$, for $\mathrm{i}=1, \ldots$, v. $\mathrm{a}\left(\mathrm{y}_{\mathrm{j}}\right)=\mathrm{v}+\mathrm{j}$, for $\mathrm{j}=1, \ldots, \mathrm{w}$.

Let $A$ and $B$ be the adjacency matrices of $G_{1}$ and $G_{2}$, respectively. Create a new adjacency matrix $\mathrm{C}$ with order $(v+w) \times(v+w)$

$$
\text { such that } C=\left(\begin{array}{ll}
A & 0 \\
0 & B
\end{array}\right) \text {. }
$$

Matrix C contains several empty skew-diagonal line bands in the middle. If we put " 1 " elements in every skew-diagonal line of the set of these empty skew-diagonal bands and make the matrix symmetric, then we obtain a EAV graph with $\mathrm{v}+\mathrm{w}$ vertices. Complete the edge labels then we have an RSEM graph $\mathrm{C}$ with order $\mathrm{v}+\mathrm{w}$.

We already knew how to generate a bigger order RSEM graph from given RSEM graphs. On the other hand, we can also generate a smaller maximal (respectively, non-maximal) RSEM graph by deleting $\mathrm{k}$ vertices (and edges incident with those vertices) of a maximal (respectively, non-maximal) RSEM graph $\mathrm{G}$ to obtain a RSEM sub graph $G^{\prime}$. However, we can only delete vertices that have the following properties:

- the k-largest labeled vertices, or

- the k-smallest labeled vertices, or

- the 1-largest labeled vertices and the $(\mathrm{k}-1)$-smallest labeled vertices.

Note that $l \leq k \leq v$. This requirement keeps the d-band set of the adjacency matrix of such graphs preserved to be a set of consecutive integers. The sub graph $\mathrm{G}$ has $\mathrm{v}-\mathrm{k}$ vertices. Note that, if we use either of the last two options, then we not only have to relabel the edges, but we also have to re-label the vertices by

- $\alpha^{*}\left(v_{i}\right)=\alpha\left(v_{i}\right)-k$ for the second option,

- $\alpha^{*}\left(v_{i}\right)=\alpha\left(v_{i}\right)-(k-1)$ for the third option.

Thus, we have the following observation.

\section{Observation 2:-}

Every SEMT graph with order at least 3 contains a smaller RSEM sub graph.

\section{Conclusion}

As mentioned in the introduction section, there are many results in RSEM labeling. However, many interesting problems remain unsolved. Here we list just a few.

- Are all trees RSEM graphs? (Conjecture from Enomoto et al. [2]).
- Can we use adjacency matrix to obtain all path-like trees? (Note that path-like tree is a tree that is derived from a path by moving some edges [8]).

- Can we find a relationship between RSEM labeling and other labeling using adjacency matrices?

- Can we use the algebraic properties of the adjacency matrix to find new properties of RSEM graph?

- Find RSEM labeling for various families of graphs.

- Find RSEM labeling by utilizing the properties of decomposition of graphs.

\section{References}

[1] M. Baca, F. Bertault, J.A. MacDougall, M. Miller, R. Simanjuntak and Slamin, Vertex antimagic totallabelings of graphs, Discussiones Mathematicae Graph Theory, 23 (2003),67-83.

[2] H. Enomoto, A. S. Llado,T. Nakamigawa and G. Ringel, Super edge magic graph SUT J. Math. 2 (1998), 105-109.

[3] Y. Fukuchi, Edge-magic labelings of Generalised Petersen graphs P(n,2), Ars Combin.,59 (2001) 253-257.

[4] J.A. Gallian, A dynamic survey of graph labeling, Electronic J. Combinatorics 5 (2003), \#DS6.

[5] Kotzig and A. Rosa, Magic valuations of finite graphs. Canad. Math. Bull. 13 (1970), 451-461.

[6] Kotzig and A. Rosa, Magic valuations of complete graphs, Publ. CRM, 175 (1972).

[7] F.A. Muntaner-Batle, Magic graphs, $\mathrm{PhD}$ Thesis, Universita Politecnica de Catalunya, Spain (2001).

[8] F.A. Muntaner-Batle, Path-like tree, personal communication.

[9] J.A MacDougall and W. Wallis, Strong edge magic graphs of maximum size.

[10] S. ShariefBasha , K. Madhusudhan Reddy \& MD.Shakeel, Algorithm To Construct Reverse Super Vertex Magic Labeling Of Complete Graphs, Global Journal of Pure and Applied Mathematics, Volume 11, Number 1 (2015), pp. 1-7.

[11] Slamin, M. Baca, Y. Lin, M. Miller and R. Simanjuntak, Edgemagic totallabelings of wheels, fans and friendship graphs.

[12] K. A. Sugeng and M. Miller, Relationship between adjacency matrices and super $(\mathrm{a}, \mathrm{d})$ edge antimagic total labeling of graphs, JCMCC.

[13] S.VenkataRamana ,S.Shareefhbasha, Reverse super vertex-magic labeling of a graph, Ph.D. Thesis. 